\title{
SIMULATION OF AIR ENTRAPMENT AND RESIN CURING DURING MANUFACTURING OF COMPOSITE CAB FRONT BY RESIN TRANSFER MOULDING PROCESS
}

\begin{abstract}
Mould filling and subsequent curing are the significant processing stages involved in the production of a composite component through Resin Transfer Moulding (RTM) fabrication technique. Dry spot formation and air entrapment during filling stage caused by improper design of filling conditions and locations that lead to undesired filling patterns resulting in defective RTM parts. Proper placement of inlet ports and exit vents as well as by adjustment of filling conditions can alleviate the problems during the mould filling stage. The temperature profile used to polymerize the resin must be carefully chosen to reduce the cure time. Instead of trial and error methods that are expensive, time consuming, and non-optimal, we propose a simulation-based optimization strategy for a composite cab front component to reduce the air entrapment and cure stage optimization. In order to be effective, the optimization strategy requires an accurate simulation of the process utilizing submodels to describe the raw material characteristics. Cure reaction kinetics and chemo-rheology were the submodels developed empirically for an unsaturated polyester resin using experimental data. The simulations were performed using commercial software PAM RTM 2008, developed by ESI Technologies. Simulation results show that the use of increase in injection pressure at the inlet filling conditions greatly reduce the air entrapped. For the cab front, the alteration of injection pressure with proper timing of vent opening reduced the air entrapped during mould filling stage. Similarly, the curing simulation results show that the use of higher mould temperatures effectively decreases the cure time as expected.

Keywords: Resin Transfer Moulding, Mould Filling Simulations, Air Entrapment, Resin Curing, Process Optimization
\end{abstract}

\section{Introduction}

Resin transfer moulding is one of the competitive manufacturing technology for producing complex and large composite structures. Development of RTM manufacturing technique for a particular application requires a proper mould design, a successful injection strategy and a well defined resin cure cycle. Determining an effective injection strategy and optimization of RTM process parameters by experimental trial and error is not practical because the retooling is expensive, time inefficient and may leads to sub-optimum solution. Hence, the virtual prototyping obtained from computer simulation of the RTM process has become the alternative cost-effective method for predicting the process performance and final quality of composite structures. RTM process consists of three phases: fiber preforming, mould filling and the resin cure cycle. The mould filling and the cure phase are considered independent, since the characteristics of the RTM flow process such as resin viscosity, applied pressure or flow rate at the injection ports, port and vent locations are usually preferred in such a way that the mould filling phase is completed before the resin gelation and the curing phase starts after the mould filling phase is completed. In other words, resin properties such as, resin gel time, resin viscosity and resin cure behaviour are the prerequisites for the prosperous RTM mould fill and cure phase simulations. A key possible problem occur during resin impregnation stage is the air entrapment, which leads to mechanical softening, early failure, or part rejection as a result of residual high void content. During mould filling process, two scales of flow occur simultaneously in the fibrous reinforcement: macroscopic flow and microscopic flow. Microscopic flow occurs between the fibre bundle and macroscopic flow occurs within the fibre bundles, as shown in Figure 1. The macroscopic flow is governed by the overall pressure gradient inside the mould. The microscopic flow is dominated by the capillary pressure which depends on the surface properties. Macroscopic flow leads to mould filling and microscopic flow impregnates the fibre preform. The in-homogeneities in the dual flows cause the formation of voids during mould filling process. Macro-voids or dry spots and micro-voids are the most experienced defective type encountered. Macro-voids are formed as a cause of macroscopic flow front in-homogeneities, where the resin flow front reaches the vent before wetting the entire preform. Macroscopic flow in-homogeneities are essentially due to either the wrong choice of inlet and vent positions of the mould which leads to flow fronts coalescence or to the presence of easy flow paths. A solution based on proper gate and vent position-

\footnotetext{
NTITUTE OF TECHNOLOGY, MESRA : RANCHI, JHARKHAND, INDIA

** INDIAN INSTITUTE OF TECHNOLOGY, KHARAGPUR, WEST BENGAL, INDIA

\# Corresponding author: raghuchemraj@gmail.com
} 


\section{Cab front design and discretization}

ing is preferable to eliminate macro-voids during mould filling. Micro-voids are formed due to the air entrapped as a result of inadequate degassing caused by the competitive flow between macroscopic and microscopic flow front, since the flow fronts at the two scales may not proceed coincidentally between fibre tows and the bulk preform.

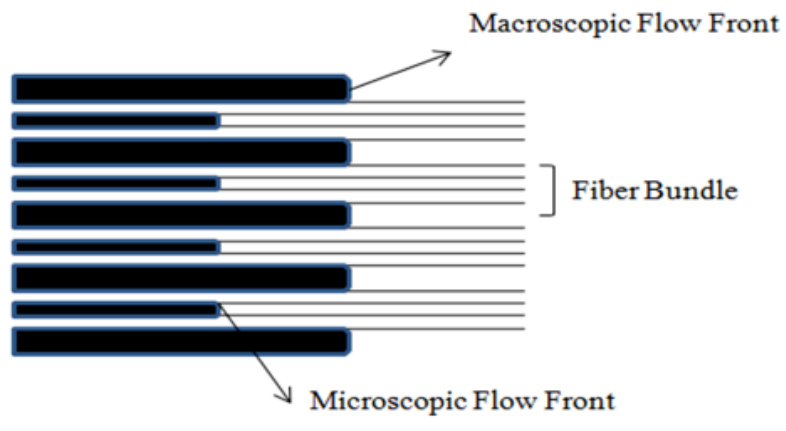

Fig. 1. Microscopic and Macroscopic Flow Fronts

Researchers have studied the use of mould filling simulation tools $[1,2]$ that predict the filling pattern based on the specification of the inlet locations and conditions as well as the prescribed permeability field of the fibre mats. The predicted filling pattern reveals the Last Point to Fill (LPF) and the locations where the air can entrap. Air entrapment can be alleviated by proper placement of inlet ports and exit vents as well as by adjustment of inlet conditions during mould filling. A number of studies have been posted on strategies of placing and timing the opening and closing of inlet ports and vents and the alteration of injection pressure to reduce or eliminate the air entrapped [3-5]

Spatial and temporal gradients of temperature and degree of cure contribute to the evolution of process-induced residual stresses, which affect the quality of the final product. Lower curing temperatures and longer processing times can produce stronger, better quality laminates but at the expense of higher processing costs [6]. There are few works in the cure process simulation such as 1-D flat plate using an implicit finite difference method [7], 2-D thick anisotropic thermosetting composites using the finite difference method [8], nonlinear transient heat transfer finite element model [9], 3-D coupled thermo-chemovisco elastic model [10].

The main objectives of this work were to develop an effective mould filling with no air entrapped and optimized cure cycle for the high speed cab front. This exercise aimed at the elimination of entrapped air using injection pressure and the optimization of cure with elevated temperatures. In this article, first the design for cab front with its dimensions and the details of component mesh are presented. In the following section, the modelling of mould fill and cure phase processes are given. In the next section, the characteristic properties of the resin matrix and the reinforcement mat used in mould fill and cure phase simulations are report. In the subsequent section, the virtual simulations of mould filling process in effort to reduce entrapped air are discussed. Finally, the optimization of cure cycle based on virtual simulation is detailed.
The cab front used by Indian Railways, which is large and complex in geometry and currently being manufactured by HLU, was chosen as the object of this study The schematic of the cab front, which is shown in Fig. 2, is of complex shape with $12 \mathrm{~mm}$ uniform thickness and approximately $3.5 \mathrm{~m}$ width and $3.0 \mathrm{~m}$ height.

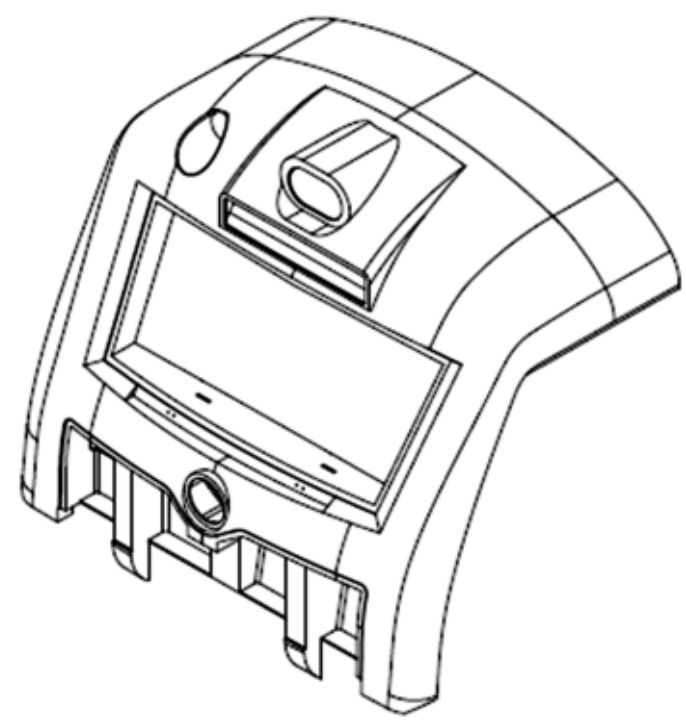

Fig. 2. Schematic of the Cab Front [11]

The mould filling in the cab front geometry was modelled as two-dimensional flow, since the thickness used is much smaller compared to the height and the width. The model was meshed with geometry meshing software Visual Mesh. Due to the complex shape of the mould, unstructured meshes composed of triangles are usually preferred and, hence the geometry was meshed with 2D triangular elements with an element size of $0.06 \mathrm{~m}$ which are shown in Fig. 3. Number of nodes and elements formed after meshing were 8084 and 15617, respectively. Further reduction in element size increases the number of nodes and elements which in turn increases the load on computation without significant improvement in the simulation results.

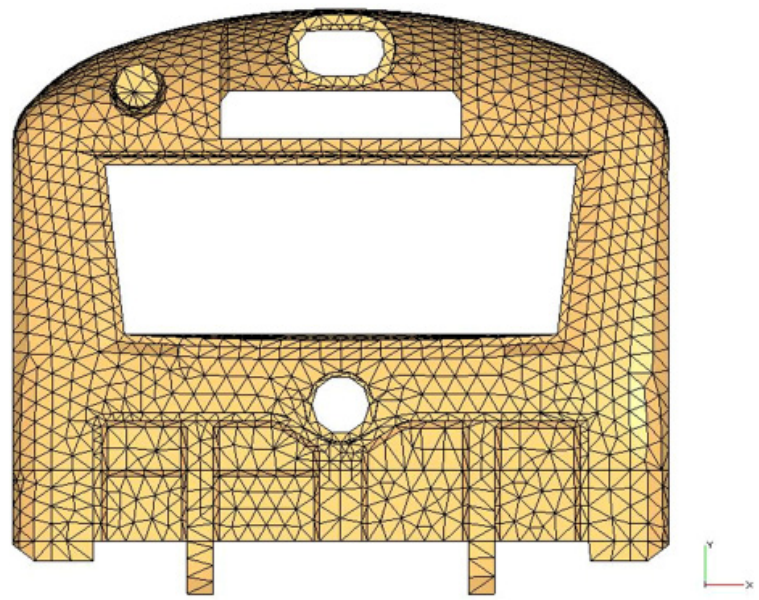

Fig. 3. Discretized Cab Front [11] 


\section{Resin flow model}

The RTM process is modelled as an incompressible fluid flow through porous medium. Darcy's law is the fundamental equation describing the flow through porous media which is given in Eq. 1.

$$
\vec{v}=\frac{\overline{\bar{k}}}{\mu} \cdot \nabla P
$$

where $\vec{v}$ is the velocity vector, $\overline{\bar{k}}$ is the permeability tensor, $\mu$ is the resin viscosity and $\nabla P$ is the pressure gradient as given in Eq. 2 .

$$
\nabla P=P_{i n j .}-P_{\text {vent }}
$$

The continuity equation for preserving the balance of resin mass is given by,

$$
\nabla \cdot \vec{V}
$$

The boundary conditions associated to solve the pressure field given by Eq. 1 are shown in Fig. 4 and given in Eqs. 4-6.

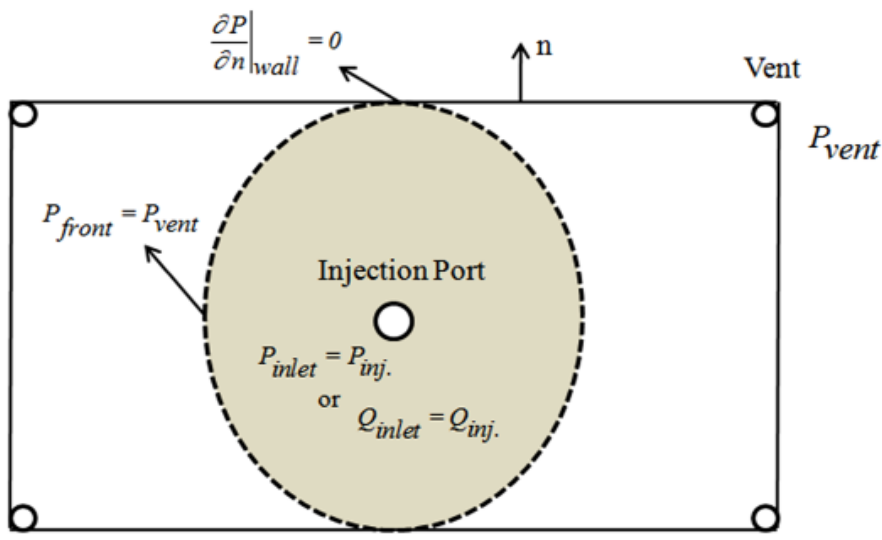

Fig. 4. Boundary Conditions for Resin Flow in a Mould [12]

At the injection port: $\quad P_{\text {inlet }}=P_{i n j .}$ or $Q_{\text {inlet }}=Q_{i n j .}$

At the flow front:

$$
P_{\text {front }}=P_{\text {vent }}
$$

At the mould wall:

$$
\left.\frac{\partial P}{\partial n}\right|_{\text {wall }}=0
$$

Where $P_{i n j .}$ is the pressure of injection, $Q_{i n j .}$ is the flow rate of the injection, $P_{\text {front }}$ is the pressure at the flow front and $P_{\text {vent }}$ is the vent pressure.

\section{Air entrapment model}

Darcy's equation as given by the form 1 is used to detect the entrapped air during mould filling simulations, but the pressure gradient is quantified with the difference between the injection pressure and the capillary pressure, as given in Eq. 7.

$$
\nabla P=P_{i n j .}-P_{\text {cap } .}
$$

where $P_{\text {cap. }}$ is the capillary pressure.
For such air entrapment cases, the pressure boundary condition at the flow front changes to the form as described in Equation 8

$$
\frac{P_{\text {void }} * V_{\text {void }}}{T_{\text {void }}}=\text { Constant }
$$

where $P_{\text {void, }}, V_{\text {void }}$ and $T_{\text {void }}$ are the pressure, volume and temperature of the entrapped air, respectively.

\section{Resin cure model}

In an applied isothermal resin cure, the rate at which heat energy is generated in a resin saturated preform is directly proportional to the rate of the cure reaction and the energy balance equation deduces to the form given by Eq. 9 .

$$
\frac{d H}{d t}=\rho_{r} * V r * H t * \frac{d \alpha}{d t}
$$

where $d H / d t$ is the rate at which the heat is generated, $\rho_{r}$ is the density of the resin, $V_{r}$ is the volume fraction of the resin, $H_{t}$ is the total heat of cure reaction, $d \alpha / d t$ is the rate of cure and $\alpha$ is the degree of cure.

The rate of cure of the resin $d \alpha / d t$, is modelled according to the empirical autocatalytic mode to describe the free-radical polymerization reaction, as shown in Equation (10)

$$
\frac{d \alpha}{d t}=k \alpha^{m}(1-\alpha)^{n}
$$

where $k$ is the cure rate constant defined by an Arrhenius expression given by Eq. 11 and $m$ and $n$ are the reaction orders of the cure kinetic model given by Eq. 10 .

$$
k(T)=A^{*} \exp ^{-(E / R T)}
$$

where $E$ is the activation energy, $A$ is the pre-exponential factor, $R$ is the gas constant $(8.314 \mathrm{~J} / \mathrm{mol} \mathrm{K})$ and $T$ is the absolute temperature $(K)$.

\section{Raw material parameters}

Medium reactive unsaturated polyester resin specially formulated for RTM process and chopped strand mat of E-class glass fibre were the raw materials considered in this study. The characteristic properties of the polymer resin matrix and the chopped strand mat used in mould filling and cure phase simulations are given in Table 1. Characteristic properties such as density, gel time, viscosity and cure kinetics of a commercially available medium reactive unsaturated polyester resin were experimentally measured. The gel time test was performed by following ASTM D2471. Viscosity at constant shear rate of $60 \mathrm{~s}^{-1}$ and at a temperature of $25^{\circ} \mathrm{C}$ was measured using Bohlin Viscometer. Differential Scanning Calorimetry (DSC) by isothermal mode was utilized to read the cure behaviour of the resin. To select suitable temperatures for the required isothermal experiments, a dynamic DSC run at a heating rate $10^{\circ} \mathrm{C} / \mathrm{min}$ was first per- 
Properties of Raw Materials

\begin{tabular}{|c|c|c|c|c|c|}
\hline \multicolumn{3}{|c|}{ Matrix Properties } & \multicolumn{3}{|c|}{ Reinforcement Properties } \\
\hline Property & Value & Unit & Property & Value & Unit \\
\hline Resin Density & 1100 & $\mathrm{Kg} / \mathrm{m}^{3}$ & Fibre density & 2540 & $\mathrm{Kg} / \mathrm{m}^{3}$ \\
\hline Raw resin viscosity & 0.6 & Pa.s & Porosity & 0.498 & No unit \\
\hline \multicolumn{3}{|c|}{$\begin{array}{l}\text { Resin gel time } \\
\text { r: Catalyst, Volume proportion) - } 52 \text { Minutes }\end{array}$} & $\begin{array}{c}\text { Permeability, } K_{x} \\
=K_{y}\end{array}$ & $9.987 * 10^{-9}$ & $\mathrm{~m}^{2}$ \\
\hline & Kineti & $\frac{d \alpha}{d t}=3.71 \times 10^{6} \exp (-$ & $\alpha^{0.092}(1-\alpha)^{1.272}$ & [13] & \\
\hline
\end{tabular}

formed. Temperatures above but near the onset of reaction were chosen. In this cure kinetics modeling, isothermal experiments were carried out at 100,110 and $120^{\circ} \mathrm{C}$. The permeability data of chopped strand mat has been obtained by conducting experiments using a constant flow 2D-radial injection set up with water as the test fluid.

\section{Simulation results}

In this work, air entrapment detection and cure process optimization were obtained using computer simulations aided by commercial software PAM RTM 2008, developed by ESI Technologies.

\subsection{Air Entrapment}

Macro-voids or dry spots and micro-voids are the most experienced defect type during mould filling stage of RTM process. Macro-voids are formed as a cause of flow front inhomogeneities, where the resin flow front reaches the vent before the entire preform has been wetted. Gross flow in-homogeneities are essentially due to either the wrong choice of inlet and vent positions or to the presence of easy flow paths. A solution based on proper gate and vent positioning is preferable to eliminate macro-voids during mould filling. Micro-voids are formed due to air entrapped as a result of inadequate degassing caused by dual scale resin flow between the fibre tows and the bulk preform. In such cases, the capillary pressure can influence the time to saturate a fibre tow significantly and impact the overall impregnation dynamics. In this work, we propose a simulation based solution that can predict and eliminate the air entrapped. Prior to this work, the optimization of inlet and vent location requiring minimum mould fill time without dry spots (macro-voids) was completed by trial and error isothermal mould filling simulations. For all the simulation trials, $10 \mathrm{~mm}$ diameter was used for injection ports and outlet vents to fill the large complex mould measuring an approximate capacity of $0.085 \mathrm{~m}^{3}$. The simulation results showed that the injection strategy of four injection points on the front face and four vents at the corners of the cab front with constant pressure of 5 atmospheres delivered an optimum mould fill time of 32 minutes without dry spots.
The entrapment of air during resin impregnation due to capillary pressure of fibre tows leads to residual high void content. The higher void content makes the part mechanically soften, early failure, or part rejection. The detection of air entrapped using flow simulations is made possible by modeling capillary pressure in each fibre tows. Ideally, the modeling of capillary pressure is accomplished by imposing a pressure value at the end of each fibre. But, the assigning of capillary pressure value at each fibre end is not practically possible for the meshed geometry. Hence, a positive pressure is maintained at the gate to impose the capillary pressure at the fibre tows.

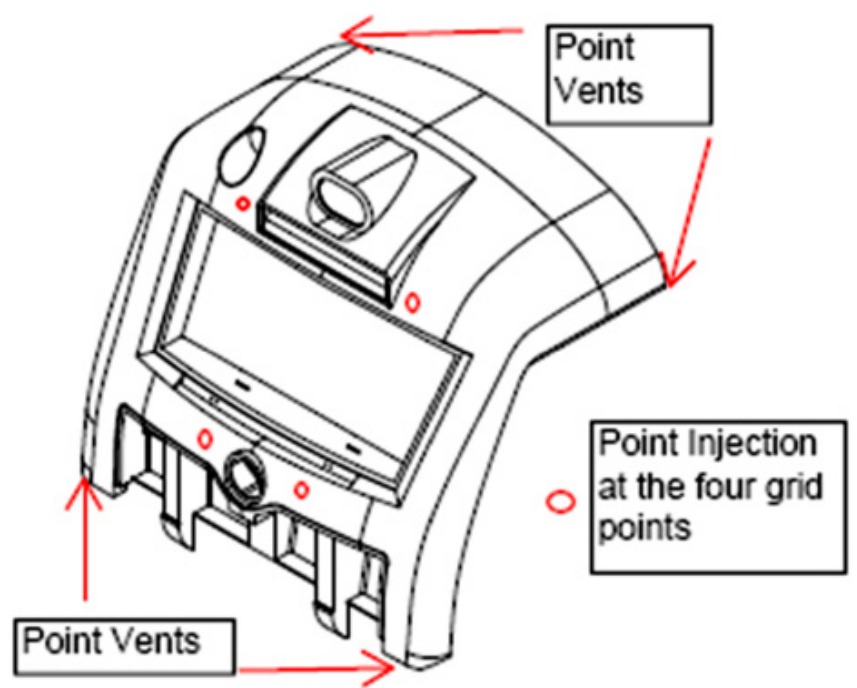

Fig. 5. Injection Strategy [11]

The cab front model using effective injection configuration was simulated to detect the entrapped air by imposing a vent pressure of $1 \mathrm{~atm}$ at all four vents placed at all the four corners. The injection pressure was kept at 5 atm in all four injection gates. The predicted simulation results shows that micro-voids are formed due to the entrapment of air towards bottom part and the middle portion of the cab front, as shown in Fig. 6. From the same Figure, it can be mentioned that the voids are formed at the junction of four flow fronts from the four injection points. Efforts have been made using alteration of injection pressure, vent opening operations to reduce the entrapped air. Using injection pressure of 6 atmospheres and all vent pressure 1 atmosphere until 10 minutes has deduced the air entrapment volume to $1 \%$ to 

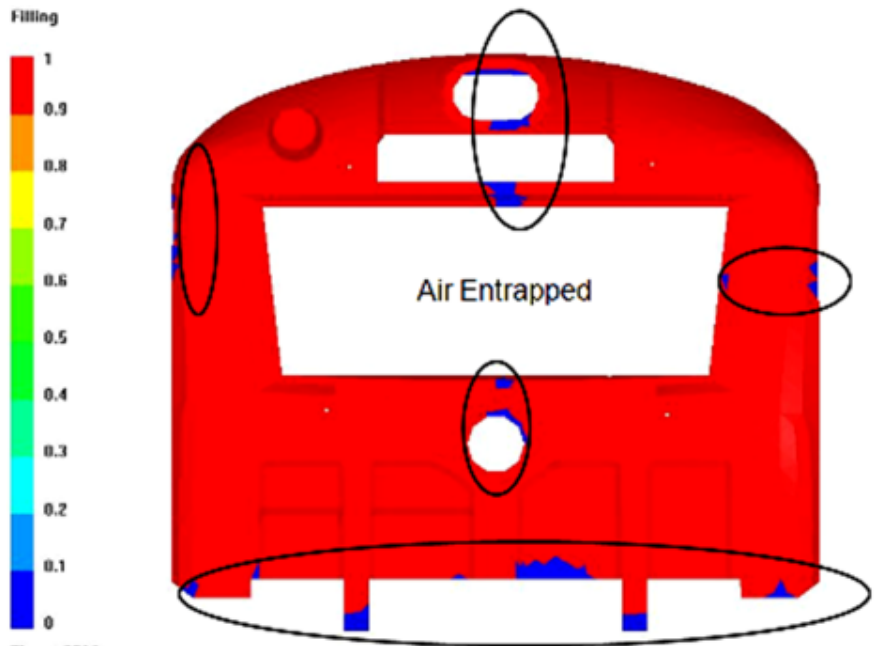

Time : 2511 \%

Fig. 6. Air Entrapment

$0.5 \%$ when compared to the volume of the cab front. The other method is to impose extra air expelling taps at the predicted air entrapped place. The vents are closed after mould filling phase and the resin is made to purge through air relieving taps.

\subsection{Resin Curing Simulations}

The objective in the cure cycle design of a RTM manufacturing process is how to set-up the time-temperature profile in such a way that certain criteria are fulfilled and the result is optimal. The formulation of the objectives should include several performance criteria for the production system such as the targeted degree of cure, the targeted maximum temperature of the part and the duration of the cure cycle as well as the production system limitations (constraints) such as the maximum allowable heating rate, the maximum allowable cooling rate etc. In most RTM cases when the main cure cycle starts, the filling stage has been concluded already. There will not be resin flow and hence, the heat transport phenomena at the other two coordinates $(x-y)$ are negligible and the corresponding heat convection terms can be omitted.

TABLE 2

Cure Simulation Results

\begin{tabular}{|c|c|c|c|c|}
\hline \hline $\begin{array}{c}\text { Sl. } \\
\text { No. }\end{array}$ & $\begin{array}{c}\text { Temperature, } \\
\text { Kelvin }\end{array}$ & $\begin{array}{c}\text { Maximum } \\
\text { Degree of } \\
\text { Cure }\end{array}$ & $\begin{array}{c}\text { Cure Time, } \\
\text { Minutes }\end{array}$ & $\begin{array}{c}\text { Peak } \\
\text { Exothermy, } \\
\text { J/Kg. }\end{array}$ \\
\hline 1 & 340 & 0.713 & 60 & 8000 \\
\hline 2 & 350 & 0.884 & 60 & 15600 \\
\hline 3 & 360 & 0.966 & 60 & 25100 \\
\hline 4 & 370 & 0.992 & 60 & 30000 \\
\hline 5 & 380 & 0.998 & 60 & 36700 \\
\hline 6 & 390 & 1 & 55 & 34100 \\
\hline
\end{tabular}

In this work, the main objective is to obtain a maximum cure within a period of 1 hour using applied elevated temperatures under isothermal conditions. The experimentally determined autocatalytic cure kinetics was used to simulate the resin cure at different temperatures under isothermal conditions. Temperature ranges from $340 \mathrm{~K}$ to $390 \mathrm{~K}$ were considered for the isothermal cure of the cab front. A constant temperature model was applied to the cab front and the degree of cure and exothermy as a function of time were extracted. The results of curing simulation are tabulated in Table 2.

The simulated degree of cure and exothermic heat flow as a function of time at different isothermal temperature applied are shown in Figs. 7,8. The composite part can be cured a wide

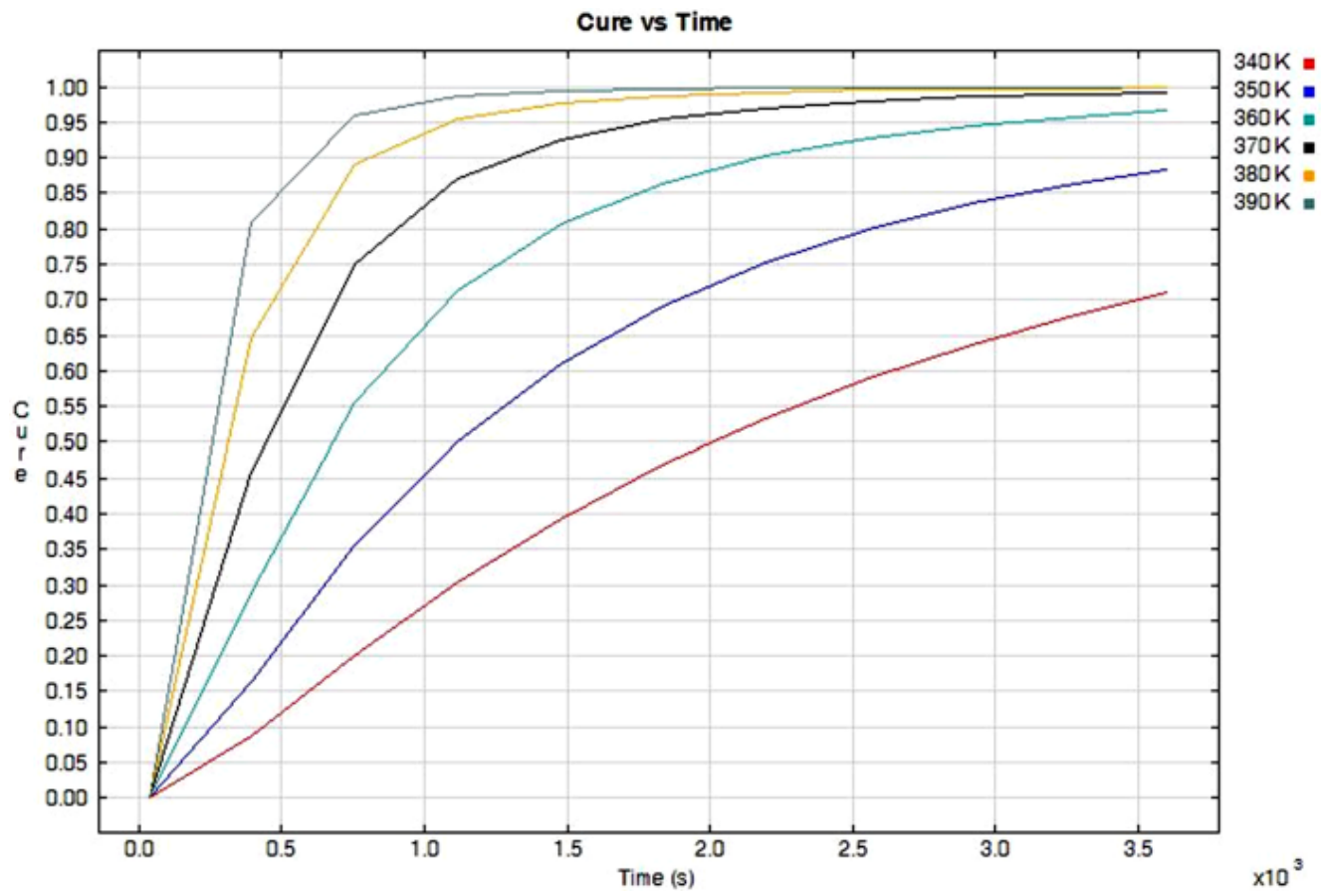

Fig. 7. Simulated Degree of Cure as a Function of Time at Different Isothermal Temperatures 


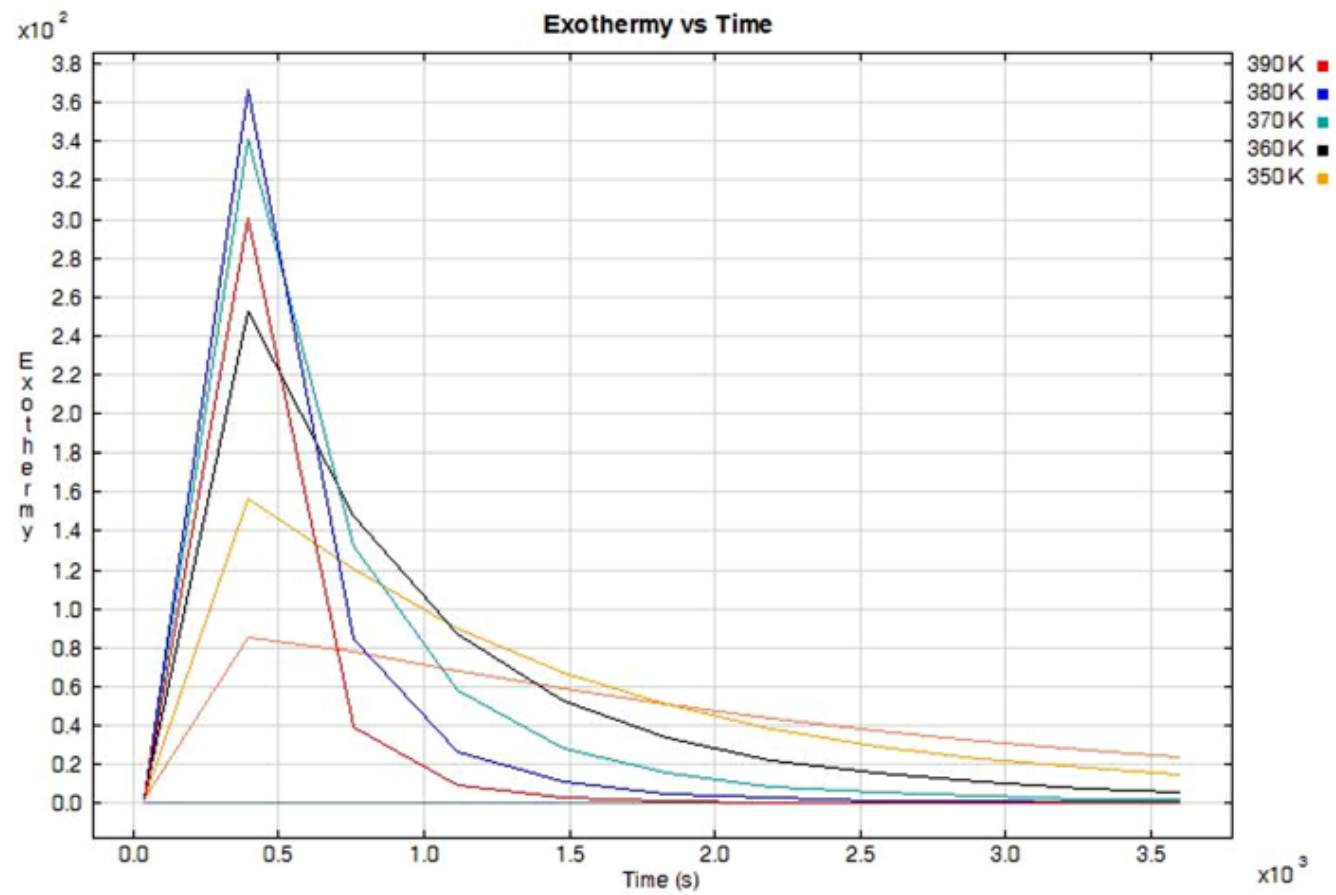

Fig. 8. Simulated Exothermic Heat Flow as a Function of Time at Different Isothermal Temperatures

temperature range. However, the selection of temperature for the part cure depends on the resin degradation temperature and the processing ease. Moreover, isothermal curing is most preferred to have a uniform cure throughout the product. From Table 2, Figs. 7,8 it is evident that the use of higher temperatures has decreased the time to obtain the maximum cure rate within applied 60 minutes. The isothermal curing at $390 \mathrm{~K}$ decreased the cure time to 55 minutes to attain the maximum cure. It can be seen that for any given time, higher the isothermal temperature, higher the degree of cure. The cure simulation shows that the resin system follows initiation, propagation, peaking and termination during curing as predicted by autocatalytic form. As expected, the value of maximum heat flow increases with increasing isothermal temperature. However, the total heat flow for all the applied temperatures is constant, which can be obtained from the area under the respective heat flow curve.

\section{Conclusion}

In this paper, the investigation of entrapped air during RTM mould filling process and optimization of cure time using virtual process simulation are proposed. Using injection pressure of 6 atmospheres and all vent pressure 1 atmosphere until 10 minutes has deduced the air entrapment volume to $1 \%$ to $0.5 \%$ when compared to the volume of the cab front. Similarly, the curing simulation results show that the isothermal curing at $390 \mathrm{~K}$ decreased the cure time to 55 minutes with the maximum degree of cure.

\section{REFERENCES}

[1] A. Chan, S. Hwang, Polym. Eng. Sci. 31, 1149-1156 (1991).

[2] M. Um, W. Lee, Polym. Eng. Sci. 31, 765-771 (1991).

[3] Y.F. Chen, V.R. Voller, K.A. Stelson, J. Compos. Mater. 31, 11411161 (1997).

[4] D. Nielsen, R. Pitchuman, 62, 283-298 (2002).

[5] J.M. Lawrence, P. Friede, S.G. Advani, Composites Part A: Applied Science and Manufacturing 36 (8), 1141-1161 (2005).

[6] D.J. Michaud, A.N. Beris, P.S. Dhurjati, J. Compos. Mater. 3136 (10), 1175-1200 (2001).

[7] A.C. Loos, G.S. Springer, J. Compos. Mater. 31, 17135-169 (1983).

[8] T.A. Bogetti, J.W. Gillespie Jr, J. Compos. Mater. 31, 239-273 (1991).

[9] S. Yi, H.H. Hilton, M.F. Ahmad, Computers And Structures 64, 16-21 (1997).

[10] Q. Zhu, P.H. Geubelle, M. Li, C.L. Tucker, J. Compos. Mater. 31 35 (24), 2171-2205 (2001).

[11] R.R. Pandiyan, K.G. Kundu, S. Neogi, J. Patel, J. Compos. Mater. 3144 (18), 2217-2231 (2010). 